\title{
Editorial for QShine 2014 Special Issue
}

\author{
Victor C. M. Leung ${ }^{1} \cdot$ Jiangchuan $\mathrm{Liu}^{2}$ • Edith $\mathrm{Ngai}^{3} \cdot \mathrm{Jianping} \mathrm{Pan}^{4}$. \\ Thanos Stouraitis ${ }^{5}$
}

Published online: 16 April 2016

(C) Springer Science+Business Media New York 2016

\section{Editorial:}

This special issue features six high quality papers, most of which have been substantially improved and extended from the best papers presented at the 10th International Conference on Heterogeneous Networking for Quality, Reliability, Security and Robustness (QShine 2014), which was held on August 18-20, 2014 in Rhodes, Greece.

The first paper "Optimal Downlink Scheduling for Heterogeneous Traffic Types in LTE-A Based on MDP and Chance-Constrained Approaches" was written by Samira Niafar, Xiaoqi Tan, and Danny H. K. Tsang. In the fast growing mobile broadband market nowadays, the high traffic demands come from a wide range of heterogeneous applications with different quality of service (QoS) requirements. This paper considers two packet scheduling problems, taking both the long-term and short-term approaches, to support QoS in LTE-A system. The paper

Victor C. M. Leung

vleung@ece.ubc.ca

1 Department of Electrical and Computer Engineering, The University of British Columbia, Vancouver, BC, Canada

2 School of Computing Science, Simon Fraser University, Burnaby, BC, Canada

3 Department of Information Technology, Uppsala University, Uppsala, Sweden

4 Computer Science, University of Victoria, Victoria, BC, Canada

5 ECE Department, Khalifa University, Abu Dhabi, UAE formulates optimization problems considering both the long-term and short-term revenues for heterogeneous traffic demands, proposes packet-scheduling schemes to solve the problems, and validates the solutions via numerical simulations.

The second paper is "Understanding and Predicting Data Hotspots in Cellular Networks" from Ana Nika, Asad Ismail, Ben Y. Zhao, Sabrina Gaito, Gian Paolo Rossi, and Haitao Zheng. The authors in this paper take an empirical approach to study the unprecedented growth of mobile data usage by analyzing a large-scale dataset from a European cellular provider. The dataset contains 3 months of records of user voice and Internet data usage, involving $734 \mathrm{~K}+$ subscribers and 5327 cell sites. They analyze the data usage at both cell and individual user levels, and seek to understand key characteristics, predictability and causes of data hotspots in today's cellular networks. The study can lead to more efficient and responsive resource management and thus better QoS provision in cellular networks.

The next article "Achieving Collision-Free Communication by Time of Charge in WRSN" was written by Yuelong Tian, Peng Cheng, Liang He, Yu Gu, and Jiming Chen, which considers wireless rechargeable sensor networks. These kinds of networks support energy harvesting from wireless signals, but the simultaneous transmission may cause collisions at the MAC layer. In this paper, the authors avoid such communication collisions through proper reader movement by exploiting the differences in the time of charge among rechargeable sensor nodes. They formulate the optimization problem and prove that the complexity of the optimal solution is NP- 
hard. They propose a simple yet effective algorithm to optimize both the reader stop location and stop time for minimizing the total communication delay. Extensive simulations show that their design can largely reduce the communication delay and outperform the baseline design by at least $20 \%$.

The fourth article is "A Network Coding Scheme to Improve Throughput for IEEE 802.11 WLAN", written by Kaikai Chi, Yi-hua Zhu, Yongchao Wu, and Victor C.M. Leung. In an infrastructure WLAN, the communication between any two nodes is relayed by an access point (AP), which may become the bottleneck and severely restrict the overall throughput. Existing coding schemes consider at most one packet per data flow. To remedy the problem, this paper proposes a network coding scheme that combines multiple buffered packets in one flow into a larger packet for coding, so that the packets participating in coding have similar sizes. The paper formulates an integer programming problem and proposes an optimal packet coding scheme, which is solved by a heuristic algorithm with low time complexity.

The next article is "Efficient Multicast Association to Improve the Throughput in IEEE 802.11 WLAN" written by Dhrubajyoti Bhaumick and Sasthi C. Ghosh. This paper deals with the problem of optimal association of stations (STAs) to APs for multicast services in IEEE 802.11 WLAN. It develops a combined greedy algorithm that selects a subset of APs with minimum cardinality for a conflict-free frequency assignment. It also finds an association between the STAs and the selected APs that maximizes the data rate. Simulation results show that the proposed algorithm selects significantly less number of APs for different data rates in comparison to the well-known metrics for multicast association.

The last article is "Throughput Optimization via Association Control in Wireless LANs" from Heyi Tang, Liu Yang, Jiang Dong, Zhonghong Ou, Yong Cui, and Jianping $\mathrm{Wu}$. The authors point out that the existing RSSI-based association strategy often leads to load imbalance among APs and decreases the aggregated throughput. The different bandwidth demand of users is usually not considered in the current AP association schemes. To address this problem, the authors introduce the bandwidth demand of users as a new constraint to formulate the Joint AP Association and bandwidth Allocation (JAA) problem. They comprehensively analyze the solution space and prove the problem NP-hard. Trace-driven evaluations show that the throughput is improved about $23.1 \%$ compared to conventional schemes.

The guest editors are thankful to the authors for their great contributions and the reviewers for their efforts in reviewing the manuscripts. We also thank the Edit-in-Chief, Dr. Imrich Chlamtac for his supportive guidance during the entire process.

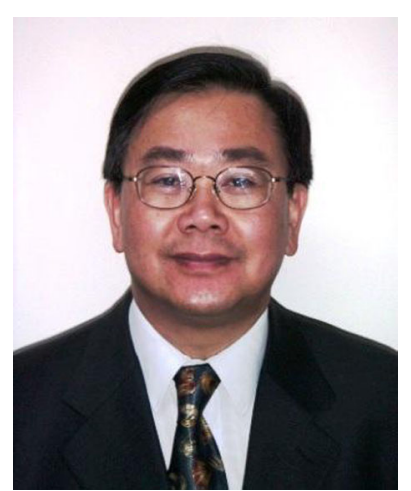

Dr. Victor C. M. Leung is a Professor and the holder of the TELUS Mobility Research Chair i $\mathrm{n} \quad \mathrm{A} \mathrm{d} \mathrm{v}$ a $\mathrm{n} \mathrm{c}$ e d Telecommunications Engineering in the Department of Electrical and Computer Engineering, the University of British Columbia. His research interests are in the areas of wireless networks and mobile systems. He has coauthored about 900 technical papers in international journals and conference proceedings, in addition to a number of edited books and book chapters in these areas. Several of his papers have been selected for best paper awards. Prof. Leung is a registered professional engineer in the Province of British Columbia, Canada. He is a Fellow of IEEE, the Royal Society of Canada, the Engineering Institute of Canada, and the Canadian Academy of Engineering. He was a Distinguished Lecturer of the IEEE Communications Society. He has served on the editorial boards of the IEEE Journal on Selected Areas in Communications - Wireless Communications Series, the IEEE Transactions on Computers, Wireless Communications, and Vehicular Technology, and currently serves on the editorial boards of the IEEE Wireless Communications Letters, IEEE Journal on Selected Areas in Communications - Series on Green Communications and Networking, IEEE Access, Computer Communications, as well as several other journals. He has guest-edited many journal special issues, and provided leadership to the technical program committees and organizing committees of many international conferences. Dr. Leung is a winner of the IEEE Vancouver Section Centennial Award and the 2011 UBC Killam Research Prize.

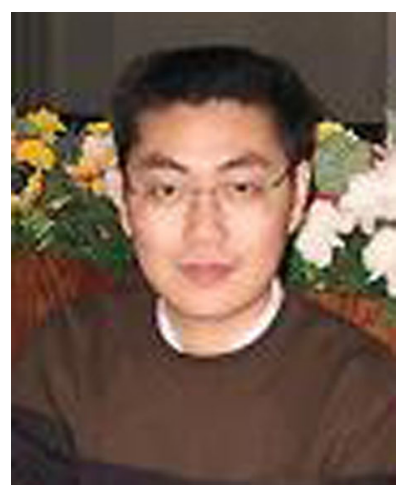

Dr. Jiangchuan Liu is a Full Professor in the School of Computing Science, Simon Fraser University, British Columbia, Canada, and an NSERC E.W.R. Steacie Memorial Fellow. He is an EMC-Endowed Visiting Chair Professor of Tsinghua University, Beijing, China (2013-2016). From 2003 to 2004, he was an Assistant Professor at The Chinese University of Hong Kong. He received the BEng degree (cum laude) from Tsinghua University, Beijing, China, in 1999, and the PhD degree from The Hong Kong University of Science and Technology in 2003, both in computer science. He is a co- 
recipient of the inaugural Test of Time Paper Award of IEEE INFOCOM (2015), ACM TOMCCAP Nicolas D. Georganas Best Paper Award (2013), ACM Multimedia Best Paper Award (2012), IEEE Globecom Best Paper Award (2011), and IEEE Communications Society Best Paper Award on Multimedia Communications (2009). His students received the Best Student Paper Award of IEEE/ACM IWQoS twice (2008 and 2012). His research interests include multimedia systems and networks, cloud computing, social networking, online gaming, big data computing, wireless sensor networks, and peer-to-peer and overlay networks. He has served on the editorial boards of IEEE Transactions on Big Data, Transactions on Multimedia, IEEE Communications Surveys and Tutorials, IEEE Access, IEEE Internet of Things Journal, Elsevier Computer Communications, and Wiley Wireless Communications and Mobile Computing.

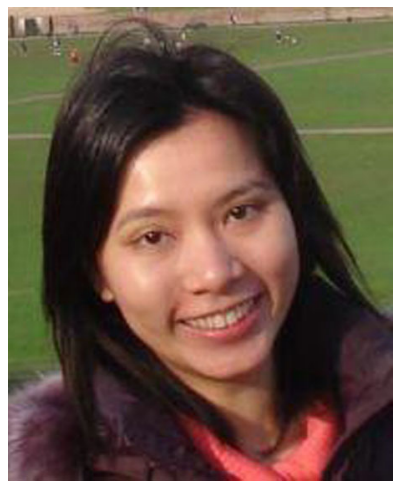

Dr. Edith Ngai is currently an Associate Professor in Department of Information Technology, Uppsala University, Sweden. She received her PhD from The Chinese University of Hong Kong in 2007. She was a postdoc in Imperial College London, United Kingdom in 2007-2008. Her research interests include wireless sensor and mobile networks, Internet-ofThings, network security and privacy, smart city and e-health applications. She is a visiting professor at Ericsson Research Sweden in 2015-2016. Previously, she has conducted research in UCLA, Simon Fraser University, and Tsinghua University. Edith is a VINNMER Fellow (2009) awarded by VINNOVA, Sweden. Her co-authored papers have received best paper runner-up awards in IEEE IWQoS 2010 and ACM/IEEE IPSN 2013. Edith has served as TPC members in major networking conferences, including IEEE ICDCS, IEEE Infocom, IEEE ICC, IEEE Globecom, IEEE/ACM IWQoS, ACM MSWiM, IEEE RTCSA, IEEE CloudCom, etc. She was a TPC co-chair of Swedish National Computer Networking Workshop (SNCNW'12) and QShine'14. She is a program chair of ACM womENcourage 2015, TPC co-chair of IEEE SmartCity 2015 and IEEE ISSNIP 2015. She has served as a guest editor in special issue of IEEE Transactions of Industrial Informatics, IEEE Internetof-Things Journal, Springer Mobile Networks and Applications (MONET), and EURASIP Journal on Wireless Communications and Networking. Edith is a senior member of IEEE and a member of ACM.

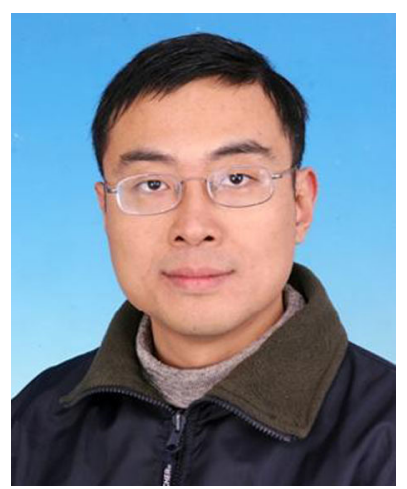

Dr Jianping Pan is currently a Professor of Computer Science at the University of Victoria, Victoria, British Columbia, Canada. He received his Bachelor's and PhD degrees in computer science from Southeast University, Nanjing, Jiangsu, China, and he did his postdoctoral research at the University of Waterloo, Waterloo, Ontario, Canada. He also worked at Fujitsu Labs and NTT Labs. His area of specialization is computer networks and distributed systems, and his current research interests include protocols for advanced networking, performance analysis of networked systems, and applied network security. He received the IEICE Best Paper Award in 2009, the Telecommunications Advancement Foundation's Telesys Award in 2010, the WCSP 2011 Best Paper Award, the IEEE Globecom 2011 Best Paper Award, the JSPS Invitation Fellowship in 2012, and the IEEE ICC 2013 Best Paper Award, and has been serving on the technical program committees of major computer communications and networking conferences including IEEE INFOCOM, ICC, Globecom, WCNC and CCNC. He is the Ad Hoc and Sensor Networking Symposium Co-Chair of IEEE Globecom 2012 and an Associate Editor of IEEE Transactions on Vehicular Technology. He is a senior member of the ACM and a senior member of the IEEE.

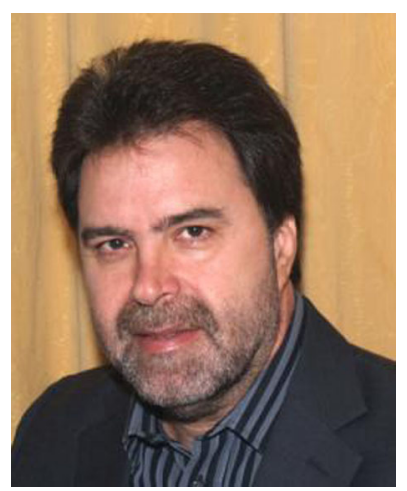

Dr. Thanos Stouraitis an IEEE Fellow for his "contributions in digital signal processing architectures and computer arithmetic," is currently Professor and Chair of the ECE department of Khalifa University, UAE (on leave from the University of Patras, Greece). He has served on the faculties of University of Florida, Ohio State University, Polytechnic University (NYU), and University of British Columbia. His research interests include cryptography, computer arithmetic, signal/image processing systems, and optimal processor design. $\mathrm{He}$ has co-authored over 180 technical papers), holds one patent on DSP processor design, and has authored several book chapters and books. He served or is serving as an Editor or/and Guest Editor for numerous technical journals. He served as general chair, TPC chair and symposium chair for many international conferences, like ISCAS, SiPS, ICECS, etc. $\mathrm{He}$ received the IEEE Circuits and Systems Society Guillemin-Cauer Award. He has served IEEE in many ways, including as CAS Society President, 2012-13. 